\title{
COVID-19: possible accelerated progression to a fatal outcome for elder patients
}

Rapid communication

José Manuel González-Rayas ${ }^{1}$

1. School of Medicine and Health Sciences, Monterrey Institute of Technology and Higher Education, Monterrey, Mexico

Keywords: coronavirus, Wuhan pneumonia, 2019-nCoV, COVID-19, SARS-CoV-2, elder, linear regression

\footnotetext{
Abstract: The demographic data available for Hubei's, China first 24 fatal cases of 2019nCoV (now SARS-CoV-2) was evaluated. The analysis revealed a weak inverse linear relation between the age of the patients and the time from case identification to death. This suggests that the progression to a fatal outcome is faster for elder patients. However, no certain conclusions can be made until more data are analyzed.

\section{Warnings:}

- This is a preliminary report, and thus, the present findings are not valid until they are confirmed with more data.

- No COVID-19 related decisions should be based on this report until it is confirmed with more data.

- The present communication should not be reported by the media until it is confirmed.

- Confirmation efforts are underway.
} 
SARS-CoV-2, 2019 novel Coronavirus or 2019-nCoV, is the causative agent of the so-called Wuhan pneumonia or COVID-19. SARS-CoV-2 has a probable zoonotic origin, with the first cases being closely related to the Huanan South China Seafood Market, in Wuhan, Hubei, China. $(1,2)$ Since SARS-CoV-2 is a new pathogen, few was initially known about it. Nonetheless, a registry comprising the sex, age, and symptoms of Hubei's first 24 fatal cases of 2019-nCoV (now COVID-19), according to China's National Health Commission $(3,4)$, was brought to light by Bloomberg and several other news agencies. (2) The registry also provides the date the patients first sought for medical help and the date of death. Additionally, it specifies which cases had a previous illness, such as diabetes, coronary artery disease, hypertension, Parkinson's disease and liver cirrhosis. Since old patients are a known vulnerable group for SARS-CoV and MERS-CoV $(1,5)$, the relation between the age of the patients infected with SARS-CoV-2 and the time elapsed from case identification to death (a variable termed in this study as "DoD" or Duration of Disease) was investigated.

When looking deeper at the dataset, it became apparent that no precise details are given about the date when case 3 (a 53-year-old male) first sought for medical help. Hence, this case was not considered. Furthermore, case 21 (a 85-year-old female) was found to have a far greater DoD when compared to other cases, making it a probable outlier. Thus, it was excluded.

The age of the patients was initially plotted against the DoD, and it became apparent that the data followed an approximate linear trend. Hence, a linear regression was performed, resulting in a Pearson correlation coefficient (r) of -0.38829 (Figure 1). Then, the registry was divided in 8 subgroups according to the sex of the patients and the presence or absence of a pre-existing illness (PEI). Subsequently, linear regressions were performed. The results, presented on Table 1, show that the linear trend appears to remain after the registry is divided according to sex and PEI. However, the later does not stand for the subgroup of male patients with a pre-existing illness, since the calculated slope of the model is positive (as opposed to the other subgroups).

The present analysis shows that there is a weak inverse linear relation between the age of patients infected with SARS-CoV-2 and the time elapsed from case recognition to death (Duration of Disease or DoD). This could indicate that old patients progress faster to a fatal outcome than younger patients. Also, the present communication is valuable, since, to the best of the author's knowledge, it is the first to provide statistical evidence of the association between DoD and age (by directly comparing the variables) for the novel Coronavirus. However, the paucity of data hampers the analysis and precludes the elaboration of further claims. To the present moment, complete demographical data (reporting the time from diagnosis to death) is limited to the first 24 fatal cases of COVID19. Thus, although deaths have far exceeded that number, it is not possible to expand the analysis to include those additional cases. Nonetheless, when the complete patient registries (including information about DoD) from the multiple countries with cases of COVID-19 are published, attempts will be directed to demonstrate the association between age and DoD for a wider age range and to obtain better $r$ coefficients. 


\section{Funding:}

No funding was received for the development of this report.

\section{Conflict of Interest/Disclosure:}

The author has declared no conflicts of interest.

\section{References:}

1. Gralinski EL, Menachery DV. Return of the Coronavirus: 2019-nCoV. Viruses 2020; 12(2): $1-8$.

2. Davies W, Tan S. The Age, Sex and Symptoms of China's Coronavirus Victims. 2020 Jan 22 [cited 2020 Jan 24]. https://www.bloomberg.com/news/articles/2020-0123/several-china-coronavirus-victims-didn-t-even-have-a-fever

3. Health Commission of Hubei Province. Hubei Provincial Health and Health Committee's report on pneumonia of new coronavirus infection [in Chinese]. 2020 Jan 24 [cited 2020 Jan 27].

http://wjw.hubei.gov.cn/fbjd/dtyw/202001/t20200124_2014626.shtml

4. National Health Committee of the People's Republic of China. Epidemic situation of new coronavirus infection on January 23 [in Chinese]. 2020 Jan 23 [cited 2020 Jan 27]. http://www.nhc.gov.cn/yjb/s3578/202001/5d19a4f6d3154b9fae328918ed2e3c8a.shtml

5. Aly M, Elrobh M, Alzayer M, et al. Occurrence of the Middle East Respiratory Syndrome Coronavirus (MERS-CoV) across the Gulf Corporation Council countries: Four years update. Ahmad R, editor. PLoS ONE 2017; 12(10):1-11. 
Table 1. Results of the linear regression between Age and DoD

\begin{tabular}{rrr}
\hline Group & $\begin{array}{c}\text { Number of patients } \\
\text { (n) }\end{array}$ & $\begin{array}{c}\text { Pearson correlation coefficient } \\
\text { (r) }\end{array}$ \\
\hline All patients & 22 & -0.38829 \\
Patients with PEI & 11 & -0.45818 \\
Patients without PEI & 11 & -0.40074 \\
Males with and without PEI & 15 & -0.30939 \\
Females with and without & 7 & -0.61387 \\
PEI & & \\
Males with PEI & 8 & 0.16291 \\
Females with PEI & 3 & -0.77061 \\
Males without PEI & 7 & -0.41731 \\
Females without PEI & 4 & -0.78112 \\
\hline
\end{tabular}

PEI: pre-existing illness

DoD: duration of disease 


\section{Figure:}

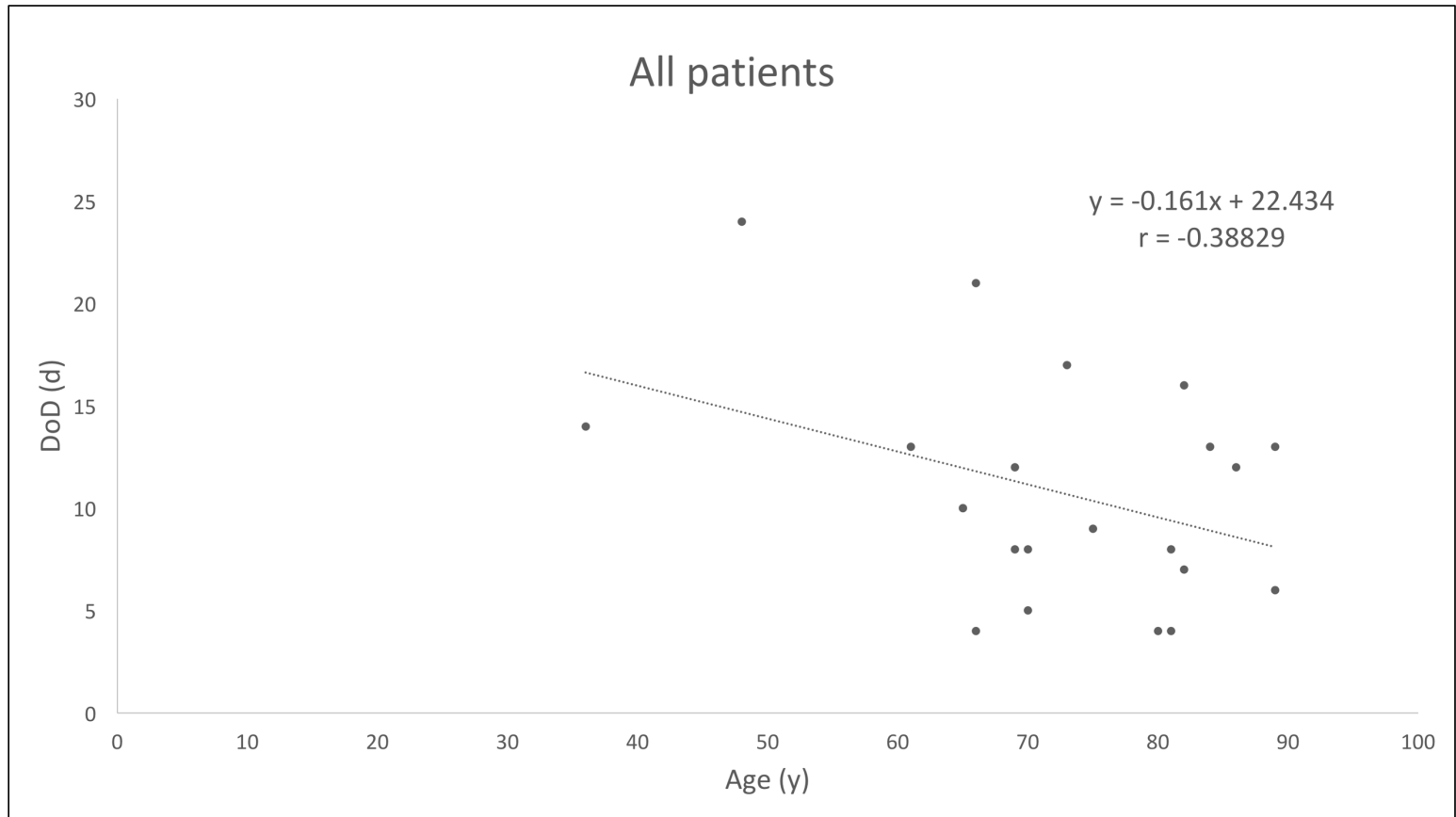

Figure 1. Scatter plot comparing the age, in years, of the patients infected with SARS-CoV2 to the time elapsed from case identification to death in days (Duration of Disease or DoD). 